in studies of the Mozart Effect. The significance of interictal epileptiform discharges and their control in the treatment of epilepsy is controversial. Nonetheless, this pilot, controlled study of the Mozart Effect, if confirmed in larger studies, could lead to new treatment interventions in epilepsy.

\title{
DAYTIME BEHAVIOR AND SLEEP DISTURBANCE IN EPILEPSY
}

Daytime behavior and sleep disturbance in 30 children with epilepsy, mean age 10.3 +/- 2.1 years, were evaluated by parent-rating questionnaires and child symptom self-report measures in a study at the University of Florida, Gainesville, FL. Complex partial seizures were diagnosed in $43 \%$ and generalized epilepsy in $57 \%$. Sixty percent were seizure-free during the 56-day test period; $83 \%$ were on anticonvulsant medications; and $20 \%$ were also taking methylphenidate, except for 72 hours prior to the sleep study. Abnormal sleep architecture, breathing abnormalities, and excessive daytime sleepiness were frequently encountered, and $24(80 \%)$ patients showed obstructive hypopneas or apneas with associated sleep disruption, as recorded by overnight polysomnograms. Parents' reports indicated significant problems with inattention/hyperactivity in children with epilepsy. Behavioral problems were related to concomitant sleep disturbance and not to the severity or type of epilepsy. Correlation analyses of sleep and behavior problems indicated a significant relation between inattention/hyperactivity on the Connors' Parent Rating Scale and REM latency $(\mathrm{P}<0.01)$ and periodic limb movement arousal (PLMA) index $(\mathrm{P}<0.05)$; between oppositional/externalizing behavior measure (Eyberg Child Behavior Inventory) and REM latency $(\mathrm{P}<0.05)$; and between the depression measure (Children's Depression Inventory) and REM latency $(\mathrm{P}<0.05)$, PLMA index $(\mathrm{P}<0.01)$, and length of apnea $(\mathrm{P}<0.01)$. An initial sleep history in children with epilepsy may reveal the need for overnight polysomnography and may aid in the diagnosis and treatment of sleep disturbances and comorbid behavioral problems. (Becker DA, Fennell EB, Carney PR. Daytime behavior and sleep disturbance in childhood epilepsy. Epilepsy Behav 2004;5:708-715). (Respond: E-mail: dbecker@hp.ufl.edu).

COMMENT. The main findings in the above study are as follows: 1) children with epilepsy frequently suffer from sleep disorders and excessive daytime sleepiness; 2) they have a significantly increased prevalence of inattention/hyperactivity; and 3) associated behavior problems are not directly related to the severity of epilepsy but rather to the concomitant sleep disturbance.

\section{Failure of sleep deprivation to increase tendency to epileptiform discharges in EEG.}

The effects of sleep, standard sleep deprivation (SSD), partial sleep-deprivation (PSD)(awake for 2 hours later the night before the EEG), and no sleep deprivation (NSD) on the odds of an epileptiform abnormality in outpatient pediatric EEGs was studied at Cincinnati Children's Hospital Medical Center, OH, during two-2-month periods. Of 820 EEGs recorded, sleep occurred in $22 \%$ of NSD, $44 \%$ of PSD, and $57 \%$ of SSD. Neither the presence of sleep nor the use of PSD or SSD protocols increased the odds of epileptiform EEGs. The authors conclude that sleep deprivation, posing a burden on both parent and child, should not be used routinely to increase the yield of pediatric EEGs. (Gilbert DL et al. 
Pediatrics September 2004;114:658-662). The authors admit that sleep or sleep-deprived EEGs may provide useful information in certain epileptic syndromes (eg electrical status epilepticus during slow sleep), and the need for sleep may vary according to the clinical indications. Since these findings are contrary to general experience and opinion of many electroencephalographers, further studies are indicated.

Melatonin in wake-sleep disorders of mentally retarded children. Melatonin in bedtime doses of $3 \mathrm{mg}$ up to $9 \mathrm{mg}$ in 25 children and young adults, aged 3.6 to 26 years (mean 10.5 years), had a significant beneficial effect on sleep latency without altering the efficacy of concomitant antiepileptic drugs. (Coppola G, et al. Brain Dev Sept 2004;26:373-376).

\section{RISK OF STATUS EPILEPTICUS IN EPILEPSY}

The occurrence of status epilepticus (SE) after the initial diagnosis of epilepsy was determined in a prospective community-based cohort study of 613 children at Montefiore Medical Center, Bronx, NY; Yale Medical School, New Haven, CT; and BIOS/NIU, DeKalb, IL. During a median follow-up of 8 years, $58(9.5 \%)$ had 1 or $>1$ episode of SE, the first occurring a median of 2.5 years after initial diagnosis (range, $<1$ month to 8.8 years). Thirty three had only one episode, 8 had 2 episodes, and 16 had 3 or more episodes. Of 56 patients with previous SE, 18 (32.1\%) had at least one further episode during follow-up compared to 40 of $557(7.2 \%)$ without history of SE $(\mathrm{P}<0.0001)$. Factors associated with a risk of SE included SE before initial diagnosis of epilepsy, younger age at onset (3 fold increased risk at age $<1$ year vs $10+$ years) and symptomatic etiology. In those with no SE before initial diagnosis, the risk of SE during follow-up was $14 \%$ in the symptomatic etiology group vs $2.6 \%$ in the idiopathic group. In those with previous SE, the relative risks for symptomatic/idiopathic groups were $52 \%$ vs $37 \%$. Overall, $13(2.1 \%)$ died, and the risk of dying was greater in children who had previous SE (5/56 [8.9\%]) vs $8 / 557$ [1.4\%] who did not $(\mathrm{P}=0.0002)$. Death in those with previous $\mathrm{SE}$ was usually associated with an underlying cause, eg neurodegenerative or encephalopathic disorder. Children who experienced SE during follow-up were less likely to be in 3 -year remission $(19.6 \%$ vs $65.3 \% ; \mathrm{P}<0.0001)$ and much more likely to have intractable seizures $(47.4 \%$ vs $8.9 \%$; $\mathrm{P}<0.0001)$. (Berg AT, Shinnar S, Testa FM, et al. Status epilepticus after the initial diagnosis of epilepsy in children. Neurology September (2 of 2) 2004;63:1027-1034). (Reprints: Dr Anne Berg, Neuroepidemiology Group, BIOS/NIU, DeKalb, IL 60115).

COMMENT. The risk of status epilepticus (SE) in children with an initial diagnosis of epilepsy is approximately $10 \%$. The risk is increased in children with a previous history of status, in younger age groups, and in those with symptomatic etiology. Data regarding antiepileptic drug levels in patients with SE would be of interest. The authors recommend abortive therapy in the home for patients at high risk of SE. A poor outcome of SE in a Netherlands study was related to inadequate AED therapy, as well as medical complications (eg respiratory insufficiency, cardiac arrhythmias), and a duration of SE greater than 4 hours (Scholtes FB, et al. Epilepsia 1995;35:1104-1112). Noncompliance with antiepileptic drug therapy or inadequate instruction regarding the use of rectal diazepam in the home were the explanations for the admission of 8 children with SE at the University Hospital of Wales, Cardiff, UK (Matthes JWA, Wallace SJ. Dev Med Child Neurol 1995;37:226-231). 